\title{
A coaching tele-consultation service to improve care for behavioral and psychological symptoms of dementia: A pilot study
}

\author{
Marie-Andrée Bruneau MD MSc FRCPC ${ }^{a, b, *}$ \\ Nathalie Bier $\mathrm{PhD}^{\mathrm{a}, \mathrm{c}}$ \\ Stéphanie Daneau RN PhD ${ }^{\text {a,d }}$ \\ Catherine Dubé $\mathrm{MSc}^{\mathrm{a}}$ \\ Laurence Villeneuve $\mathrm{PhD}^{\mathrm{e}}$ \\ Caroline Ménard $\mathrm{MA}^{\mathrm{e}}$ \\ Anne Bourbonnais RN PhD ${ }^{\mathrm{a}, \mathrm{d}}$
}

\begin{abstract}
${ }^{a}$ Centre de recherche de l'Institut universitaire de gériatrie de Montréal, Montréal, Québec, Canada; ${ }^{b}$ Department of Psychiatry, Faculty of Medicine, University of Montreal, Montréal, Québec, Canada; 'École de réadaptation, Faculté de médecine, Université de Montréal, Mon-

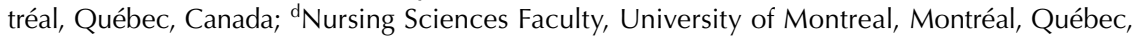

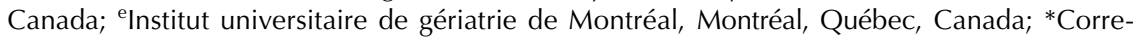
sponding author: marie.andree.bruneau@umontreal.ca
\end{abstract}

\begin{abstract}
M.-A. Bruneau, N. Bier, S. Daneau, C. Dubé, L. Villeneuve, C. Ménard, A. Bourbonnais. A coaching tele-consultation service to improve care for behavioral and psychological symptoms of dementia: A pilot study. Gerontechnology 2020;19(1):42-53; https://doi.org/10.4017/ gt.2020.19.1.005.00 Background Behavioral and psychological symptoms of dementia (BPSD) are highly prevalent in major neurocognitive disorders and have considerable impacts. Many clinical settings struggle with insufficient knowledge, suboptimal management and excessive use of psychotropic medications. Research aim Given the low availability of specialized staff and the remoteness of some healthcare locations, our aim was to document the effects and the implementation process of a telehealth coaching service on BPSD care. Methods Geriatric specialists at the Institut universitaire de gériatrie de Montréal (IUGM) offered a structured training program to distant community care teams through tele-consultation. It involved setting up a BPSD team in each participating community to ensure acquired skills would be sustained. The program proposed a 2-day formal training followed by a monthly complex BPSD case tele-consultation. One of these clinical projects with the Magdalen Islands health centre (MIHC) included a mixed methods research component. We evaluated clinical benefits by comparing Neuropsychiatric Inventory (NPI-Q), Clinical Global Impression (CGI-I) and psychotropic intake at baseline and three months post tele-consultation, on clinical chart review with non-parametric Wilcoxon analysis. The implementation process was evaluated by open-ended interviews and satisfaction questionnaires. The qualitative data were analyzed using Miles and Huberman's approach. Results Twelve patients were evaluated by tele-consultation. Results showed clinical benefits (decrease of 35\% NPI-Q ( $p=0.024)$, an improvement on CGI, as well as high satisfaction levels (caregivers and remote team). Eleven open interviews were completed (2 nurses, 3 psychosocial staff, 2 managers, 4 doctors). The project helped to define a better care trajectory for BPSD. Professionals felt a sense of competency and relief from helplessness and isolation. They also described improved patient care (systematic evaluations, individualized interventions integrating non-pharmacological aspects, more appropriate prescriptions). Conclusion These results suggest that tele-consultation is a good way to disseminate expertise concerning BPSD.
\end{abstract}

Keywords: Dementia, neurocognitive, tele-consultation, neuropsychiatric, BPSD 


\section{INTRODUCTION}

The province of Quebec encompasses 17 large regions spread over a territory of 1,667 million km2, with more than 8300000 inhabitants. Eighteen percent of Quebec's population is over 65 years old and most seniors live in remote areas distant from any major metropolis (Institut de la statistique du Québec, 2016). More than 120000 Quebecers currently suffer from Alzheimer's disease (Ministère de la Santé et des Services Sociaux [MSSS], 2016). In long-term care homes, as many as $80 \%$ of residents have a diagnosis of dementia requiring special medical care. In fact, older people with dementia suffer from many medical comorbidities and mobility difficulties that make traveling to consultations with a distant specialist a challenging task.

Behavioral and psychological symptoms of dementia (BPSD; depression, anxiety, psychosis, wandering, disinhibition, etc.) are highly predominant in Alzheimer's disease (Fernández, Gobartt, \& Balañá, 2010). Indeed, almost every patient diagnosed with this disease (80-97\%) will present behavioral or psychological symptoms (Gauthier et al., 2010). According to some estimates, psychotic symptoms can be observed in as many as $76 \%$ of $\mathrm{AD}$ patients, depressive symptoms and agitation in nearly half, and apathy in almost all patients with dementia (American Psychiatric Association, 2013). These symptoms are of great clinical importance, as they exacerbate functional and cognitive incapacity (Okura et al., 2010) and decrease patient and caregiver quality of life (Hurt et al., 2008). Physical and chemical restraints are often used in BPSD patients, with negative consequences such as the increased risk of hospitalization, early institutionalization, and increased health costs (Luppa, Luck, Brahler, Konig \& Riedel-Heller, 2008; Murman \& Colenda, 2005; Ornstein \& Gaugler, 2012).

In 2009, a committee of experts mandated by Quebec's health and social services ministry (MSSS) tabled a report (Bergman et al., 2009) that recommended developing a ministerial action plan on Alzheimer's disease and related disorders. An urgent need for action emerged from the many gaps the committee found in the services offered to people with Alzheimer's disease in Quebec in terms of specialized resources, service accessibility, continuity and quality of care. Specifically, they described a lack of knowledge and professional training as well as limited accessibility to expert care for BPSD. They also documented high levels of burden and distress among caregivers and overutilization of antipsychotic medications (prescribed to as many as a quarter to a third of dementia patients (MSSS, 2016), the highest level in Canada). Many concrete actions were taken as a result of this report. For example, training programs were established for all professionals in related fields and new clinical guidelines and tools were developed. Access to personalized coordinated care was prioritized through a collaborative chronic care model based on primary care implementation projects. Phase 2 of this plan involves disseminating this model across all regions of Quebec and consolidating memory clinics and BPSD teams. BPSD teams are interdisciplinary, composed of professionals qualified to carry out assessments and specialized interventions with seniors who have complex, persistent neuropsychiatric symptoms associated with dementia.

Given the limited availability of specialized staff in remote healthcare settings and the resulting erratic accessibility to specialized services (Rechel et al., 2016), tele-consultation has been proposed as an effective way to disseminate expertise. Many studies have shown the potential of this approach to diagnose dementia, provide follow-up (Azad, Amos, Milne, \& Power, 2012; Barth, Nickel, \& Kolominsky-Rabas, 2018; Dang, Gomez-Orozco, van Zuilen, \& Levis, 2018; Lee et al., 2000; Wadsworth et al., 2018) and offer consultations to geriatric patients with mental health issues (Dham et al., 2018; Ramos-Rios, Mateos, Lojo, Conn \& Patterson, 2012; Shulman, Conn, \& Elford, 2006). Some studies support the use of telepsychiatry in a consultation-liaison model for long-term care dementia patients (Lee et al., 2000). These studies report a high level of satisfaction among stakeholders as well as users, with no documented difference in the quality of therapeutic alliance (Azad et al., 2012). Some have shown benefits such as a decrease of symptoms (Catic et al., 2014; Lyketsos \& Roques, 2001; Steinberg, 2014), acute hospitalization rate (Catic et al., 2014; Lyketsos \& Roques, 2001; Steinberg, 2014), cost of care (Rabinowitz et al., 2010), antipsychotic prescriptions and use of physical restraints (Gordon et al., 2016). Structured support that includes coaching, with emphasis on interdisciplinary assessment and treatment adapted to the particular needs of the person, appears the most effective (Morgan et al., 2009; Saligari \& Flicker, 2002). Success factors have been identified in the literature (Loh \& Donaldson, 2007; Saligari \& Flicker, 2002) regarding the quality of the visual and auditory resolution of the equipment; appropriate screening of patients; obtaining a complete file in pre-consultation and, in particular, the availability of a trained staff at remote sites (often a pivot nurse) to prepare the consultation, perform certain cognitive or physical examinations, give comfort to the patient and his relatives, and provide medical-nursing followup after the tele-consultation. Some concerns have been reported about using tele-consultation with patients who have sensory deficits or severe 
behavioral symptoms (Dham et al., 2018).

We found a single study in the literature that reports a qualitative assessment of this type of service for BPSD. Doyle et al. (Doyle, Jackson, Loi, Malta, \& Moore, 2016) established a mentoring and consultation service in order to test a model for improving medical supervision and clinical governance to staff and clients with BPSD in regional and remote areas of Australia. They designed a qualitative and quantitative study to document the acceptance and impacts of the program by staff interviews, questionnaires and case conference evaluations.

Based on these studies, geriatric specialists at the Institut universitaire de gériatrie de Montréal (IUGM) established a tele-consultation service to coach teams in remote areas by formal teaching and monthly complex BPSD cases teleconsultations. To date, this is the only tele-consultation service offered for BPSD in Quebec. Since 2011, 9 agreements have been signed between the IUGM and regional partners. One of these partnerships, with Magdalen Islands health and social services centre (MIHC), included a formal research project. The objectives of this project were to document the effects of the service (the clinical benefits) as well as the implementation process (facilitators and barriers to the implementation of the program) of a tele-consultation and coaching service, as a way to diffuse expertise to primary caregivers, so they could eventually become autonomous and be able to manage BPSD patients without having to refer to specialized care.

\section{Methods}

This evaluative research project implied a concurrent mixed design (Creswell \& Plano Clark, 2007). We used a quantitative pre-post design for the assessment of the program effects on clinical outcomes. Because of the limited number of patients evaluated in tele-consultation and specific aspects of the MIHC clinical environment (low amount of cases, an urgent need for staff formation and only one long-term care setting), it was not possible to conduct a study with a control group. A qualitative unique case study, where a case was defined as the health care setting (MIHC), was used for the evaluation of the implementation process. The project was approved by the institutional review board and informed consent was obtained for all participants. Data from medical chart reviews were completely anonymized.

\section{Case and intervention description}

MIHC offers general clinical services to a population of over 12,000 inhabitants, of whom more than $23 \%$ are over 65 years old. The general hospital has 30 beds (4 psychiatric beds), and there are 64 long-term care beds as well as 24 beds in intermediate resources. Two general psychiatrists are on site but there is no available consultant in geriatrics, neurology or geriatric psychiatry. A one-year clinical teleservice agreement was signed between the IUGM and the MIHC team in 2016. It involved setting up a local BPSD team dedicated to the project to ensure the sustainability of the skills team members would acquire, thus becoming local experts. The service involved a preliminary in-site meeting between the teams from the IUGM (geriatric psychiatrist and geriatric psychologist) and the local BPSD team of the MIHC (two nurses and one social worker). The IUGM team visited the facilities to familiarize themselves with the care context. Thereafter, a 2-day formal training (conference on evaluation and intervention for BPSD, training in the use of clinical tools, cognitive and extrapyramidal exams, modeling with co-evaluation of 2 complex cases) was offered to the MIHC team. Afterward, a monthly complex BPSD case (chosen and prepared by the remote team) tele-consultation was organized. The teleconsultation clinics occurred at the rate of half a day per month, for a year. During this half a day, a new case was evaluated and follow-ups of previous cases were discussed as well as emergent topics brought by the MIHC team. Teleconsultation equipments were located in both hospital settings and teleconsultations were conducted through a secure and encrypted network managed by $\mathrm{CBCl}$ Telecom. This network uses the infrastructure of Integrated Network Multimedia Tele-communications, a secure high-speed fiberoptic network distinct from the Internet. Patients referred for a teleconsultation had a diagnosis of dementia and suffered from BPSD deemed as complex by the referring clinical staff because of resistance to first-line interventions.

In preparation for tele-consultation, the geriatric psychiatrist and psychologist from the IUGM reviewed the patient file transmitted by the remote team. Patients or designed surrogates signed a consent form for tele-consultation. During teleconsultation, the IUGM team members first met with the patient, a family member, a member of the direct care team and the staff member (pivot) from the MIHC's BPSD team who was responsible for the consultation. A formal assessment was conducted by the geriatric psychiatrist and geriatric psychologist during the consultation. Then the patient left, and all members of the MIHC BPSD team joined in. A summary of the problems and diagnostic impressions was followed by a joint discussion of the intervention plan. At the end of tele-consultation, the IUGM team produced a report, including various recommendations (pharmacological and non-pharmacological interventions), which was forwarded to the MIHC 
team in the following days. A follow-up was done by the geriatric psychiatrist and psychologist with the partner institution around the time of the next tele-consultation as well as 3 months later, to have an update on the patient's status. At the end of the one-year, the local team was presumed to have become autonomous in their BPSD management and tele-consultations with IUGM experts were restricted to as needed only.

\section{Data collection}

\section{Quantitative data}

Demographic and clinical characteristics of patients (age, sex, marital status, place of residence, education level, medical and psychiatric diagnosis, list of medication, last MMSE) were obtained from the patient's charts.

To assess the effects of the intervention, we measured three variables, which were obtained by the patient's chart review namely the Neuropsychiatric Inventory-Q (NPI-Q), the Clinical Global Impression-Improvement questionnaire (CGI-I) and a comparison of psychotropic medication. Each of these variables was collected before the first tele-consultation(T0) and 3 months after the first tele-consultation (T3).

The Neuropsychiatric Inventory (NPI) was developed by Cummings (1997) to assess dementia-related behavioral symptoms. It has high content validity and inter-rater reliability. The NPI examines 12 sub-domains of behavioral functioning. The Q-version documents if every 12 sub-domains is either present or absent and rates the frequency and severity of the symptoms on a 3-points scale, and caregiver distress on a 5-points scale.

The Clinical Global Impression-Improvement (CGI-I) questionnaire is a well-established research rating tool applicable to all psychiatric disorders. It is frequently used in medical care and clinical research because of its face validity and practicability. It compares the improvement of patient's condition between their admission and their current condition on a 7-point scale from $1=$ very much improved to $7=$ very much worse (Guy, 1976).

Psychotropic medication was compared from T0 to T3 by noting the presence/absence of seven classes of medication (antipsychotics, antidepressants, anticonvulsants, benzodiazepines/hypnotics, AChE Inhibitors, memantine, narcotics). The total number of medications and psychotropics prescribed were noted. Medication was revised by the geriatric psychiatrist and considered inappropriate if there was the presence of benzodiazepines/hypnotics, presence of medication with high anticholinergic side effects or presence of two or more antipsychotic medications (American Geriatrics Society, 2015).

The satisfaction with the intervention was also assessed with two satisfaction questionnaires developed by the IUGM team, inspired by other telemedicine satisfaction questionnaires (Bakken et al., 2006) and adapted to suit the needs of our population. The caregiver's questionnaire comprises 13 questions to be answered on a 5 Likert-type scale (from completely in disagreement to completely in agreement) about the referral process, technical aspects, comfort with use and satisfaction with the assessment and recommendations. The remote BPSD team satisfaction questionnaire consisted of 11 questions with the same format answered by the pivot from the MIHC BPSD team. After each tele-consultation (T0), these two satisfaction questionnaires were filled out and sent to the research team anonymously.

\section{Qualitative data}

To assess the implementation process, we used two implementation questionnaires and semistructured interviews. The implementation questionnaires were created by the IUGM team. The first questionnaire focused on the teleconsultation per se. It was completed by the IUGM team after each tele-consultation and gathered information regarding the number and type of professionals attending the tele-consultation, patient's tolerability and if there was any technical incident with tele-conferencing technology. The second questionnaire was the partner institution form and was completed by the MIHC team at T3 for the purpose of identifying through yes/ no answers if the remote team had a better understanding of BPSD, and an improved sense of competency and BPSD knowledge. It also identified if the patient's place of residence changed, if there was an emergency visit, use of physical restraints, or any side effect due to the patient's medication. Additional questions were related to the actual application of IUGM recommendations and, if they could not be implemented, what the barriers were.

Semi-structured individual interviews with different professionals from MIHC involved in the tele-consultation service were also conducted in person by a research assistant trained in qualitative research, using a flexible interview guide developed previously by the research team. Questions were constructed in order to document facilitators and barriers, worries and hopes regarding the tele-consultation project and to describe organizational and clinical changes created by the project. The interviews were audiorecorded and then transcribed by the research team. Eleven individual interviews were conducted, with an average duration of 37 minutes. 
Ten interviews were conducted in person and one by conference call.

\section{Data analysis}

Descriptive statistical analysis was used to describe patient characteristics and the results on satisfaction questionnaires. Pre-post (T0-T3) $\mathrm{NPI}-\mathrm{Q}$, and CGI-I measures were compared using non-parametrical analysis (Wilcoxon test) because of the small sample and non-normal distribution. Numbers of total medication, total and specific classes of psychotropics were compared between T0 and T3.

The data from the implementation questionnaires were aggregated in categories and results gathered and resumed. A content analysis of the qualitative data from the interviews was performed by a research assistant trained in qualitative research using Miles and Huberman's (2003) approach, i.e. condensing data, displaying similarities and differences in data, and drawing conclusions. To condense data, inductive codes were applied to the transcription to identify relevant meaning units and memos were written on methodological decisions and hypotheses. The coding convention was used to track the type of participants. Afterward, codes were organized hierarchically to display their similarities and differences and identify subthemes. The research team discussed these initial

Table 1. Patient's characteristics

\begin{tabular}{|c|c|}
\hline Demographic data & $\begin{array}{l}\text { Mean (Standard } \\
\text { Deviation) }\end{array}$ \\
\hline Age (years) & $78,5(13,2)$ \\
\hline Schooling (years) & $7,0(3,4)$ \\
\hline Women $(\% ; n)$ & $75 \% \mathrm{~N}=9$ \\
\hline 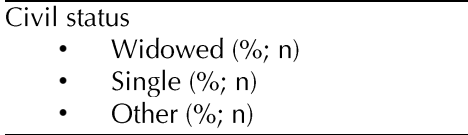 & $\begin{array}{l}42 \% \mathrm{~N}=5 \\
25 \% \mathrm{~N}=3 \\
33 \% \mathrm{~N}=4\end{array}$ \\
\hline $\begin{array}{cl}\text { Place of residence } \\
\text { - } & \text { Long term care }(\% ; n) \\
\text { - } & \text { Home }(\% ; n) \\
\text { - } & \text { Other }(\% ; n) \\
\end{array}$ & $\begin{array}{l}58 \% N=6 \\
25 \% N=3 \\
17 \% N=3\end{array}$ \\
\hline $\begin{array}{cl}\text { Type of Neurocognitive disorders } \\
\text { - } & \text { Mixed }(\%) \\
\text { - } & \text { Alzheimer's type dementia }(\%) \\
\text { - } & \text { Vascular }(\%) \\
\text { - } & \text { Other }(\%) \\
\end{array}$ & $\begin{array}{l}50 \% \\
20 \% \\
20 \% \\
10 \% \\
\end{array}$ \\
\hline $\begin{array}{l}\text { Psychiatric disorders }(\%) \\
\bullet \quad \text { Depression or anxiety }\end{array}$ & $\begin{array}{l}67 \% \\
58 \%\end{array}$ \\
\hline $\begin{array}{l}\text { Family psychiatric disorders } \\
\bullet \quad \text { Depression or anxiety }(\%)\end{array}$ & $\begin{array}{l}46 \% \\
17 \%\end{array}$ \\
\hline Number of BPSD & $3,6(2,3)$ \\
\hline $\begin{aligned} \text { BPSD } & \\
\text { - } & \text { Irritability }(\%) \\
\text { - } & \text { Aberrant motor behavior }(\%) \\
\text { - } & \text { Agitation/aggressive }(\%)\end{aligned}$ & $\begin{array}{l}67 \% \\
58 \% \\
58 \%\end{array}$ \\
\hline
\end{tabular}

sub-themes to further refine them and identify themes. Once preliminary themes were identified, the research team met to compare these qualitative results with the one from the implementation questionnaire and from the quantitative results (effects and satisfaction). This allowed furthering the interpretation of the results as a whole.

\section{Results}

Twelve tele-consultations occurred between September 2016 and September 2017. During the study, 3 patients died and their follow-up data (T3) could not be documented.

\section{Patient's characteristics}

Twelve patients were seen in tele-consultation. The majority of these were women $(75 \%)$. Patients were on average 79 years old and had 7 years of education. Almost half were widowed $(42 \%)$. More than half lived in a long-term care home $(58 \%)$. Half of the patients presented mixed dementia (50\%), 20\% Alzheimer's type of dementia or vascular disease. Most of the patients presented a past psychiatric history $(67 \%)$, mostly depression or anxiety (58\%). Almost half of the patients presented a familial psychiatric history $(46 \%)$. Patients showed on average 3.6 BPSD, mostly irritability $(67 \%)$, aberrant motor behavior $(58 \%)$ or agitation/aggressiveness (58\%) (Table 1).

\section{Clinical effects of the tele-consultation}

For the 9 patients whose follow-up was available, the NPI-Q total decreased by a mean of $35 \%$ from T0 to T3 $(p=0.024)$. Mean BPSD severity decreased by $14 \%$ and mean BPSD caregiver distress by $38 \%$ (Figure 1).

As for the CGI, clinical global improvement at 3 months was described as improved in most patients (4/9 subjects strongly improved, 3/9 slightly improved). Only 2 showed no changes in global status, but none showed a global decline in status. The total medication prescribed decreased by $18 \%$ between T0 vs T3. However, an increase of $14 \%$ of psychotropic prescriptions was observed. Comparing changes in psychotropic classes prescribed between T0 and T3 revealed an increase in antidepressant, antipsychotic and narcotic medications, and no changes in anxiolytic/sedative medications.

\section{Implementation process of the teleconsulta- tion and effects on staff}

The technological feasibility and acceptability of the tele-consultation service were demonstrated by the occurrence of only 2 technical problems: in one tele-consultation the sound was unclear, and in another, the parties involved were disconnected 3 times. Only 2 patients had difficulties tolerating tele-consultation: 1 patient showed irritability towards the questions asked, and the 


\section{Results}

$$
\text { NPI-Q ( } n=9)
$$

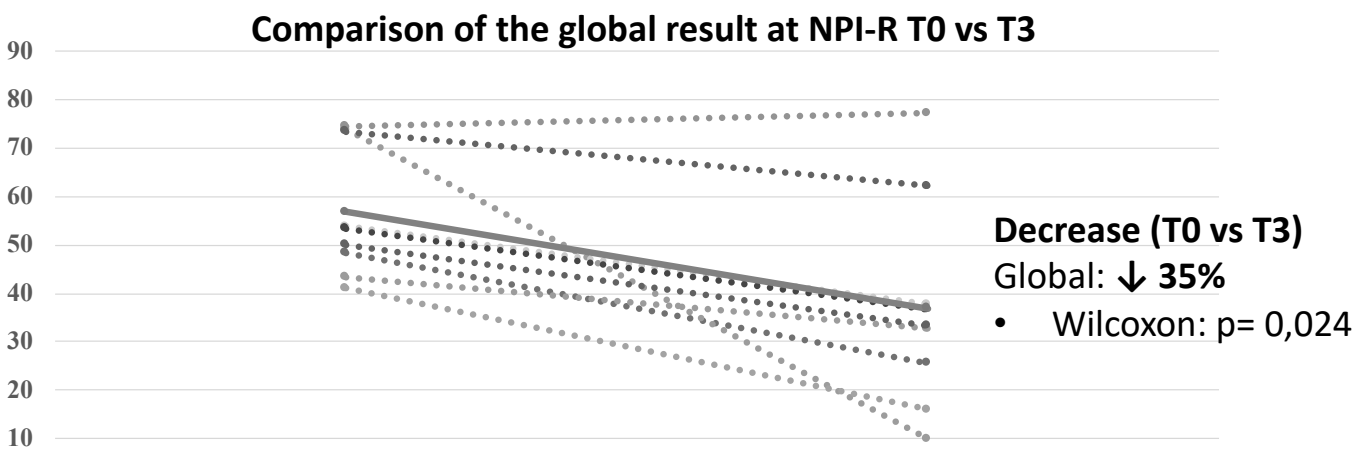

0

Figure 1. Mean of Behavioral and psychological symptoms of dementia (BPSD) severity

other experienced difficulty hearing the IUGM team due to significant deafness.

Three months after every tele-consultation, the 3 pivot professionals from MIHC BPSD team all considered that they had a better understanding of the patient's problem, had an increased sense of competency regarding BPSD and were more knowledgeable about BPSD management. They reported that all non-pharmacological recommendations were applied (100\%). On the other hand, only $63 \%$ of pharmacological recommendations were followed by medical MIHC staff. Only 1 patient had moved to a long-term care home at T3. No patient needed to be hospitalized. No use of physical restraints or side effects were reported.

Professionals from MIHC BPSD team showed a high level of satisfaction (91\%) with teleconsultations. The caregivers' satisfaction questionnaire also showed a high level of satisfaction (75\%) but denoted a slight dissatisfaction (3\%) or neutrality $(9 \%)$ towards recommending the use of this type of service to other people and to preferring to use of tele-consultation rather than to travel to meet specialists.

The feasibility and acceptability of the teleconsultation service and the effects on staff were further described during the interviews. The participants to the interviews consisted of 3 general physicians, 1 general psychiatrist, 2 clinician nurses, 2 manager nurses, 1 social worker, and 2 specialized educators. They had a mean work experience of 17 years (range from 2 to 37). Five themes emerged from these interviews.
1. Resolving isolation and helplessness by creating a team that feels better supported

Participants identified a strong impact of the tele-consultation project on the isolation they experienced when difficult behaviors occurred. The presence of the IUGM team brought great relief to the MIHC team, which often felt powerless and helpless. As a participant said:

"But you know, we do what we can. But there comes a time when we have our limits and we need to talk to someone. And to know that there is something, someone identified you know, who we can call, and that, that she knows that she can be called too. But, (...), it's for the patient, and for us, and (...) it's just positive all around."

The project also had a confirmatory effect, as professionals were able to realize that many aspects of their pre-project plan of actions were quite relevant and appropriate.

\section{More effective global management of BPSD} and positive impact on all involved

Participants expected that the project would enable them to be better equipped to intervene when patients demonstrated BPSD. Through the project, BPSD was found to be managed more effectively and more in line with established best practices, including rapid recognition, more complete and objective evaluation, and more responsive interventions for individual needs. This corroborates the quantitative results about the decrease in BPSD and decrease in caregivers' distress.

Participants also stated that they better recognized the existence of BPSD and were able to quickly identify symptoms when they occur, while these 
different problematic behaviors were previously associated with the "normal" course of the disease. In addition, the evaluation of patients with BPSD was found to be more complete and objective. Participants reported that they now knew what information to look for and what tools to use in order to assess the problem effectively, including behavioral grids and life history. Non-physician practitioners felt more confident about discussing behavior and physicians could rely on useful data to understand and reflect on the situation. The possible causes of behaviors were henceforth sought systematically. This assessment makes it possible to develop strategies that were better adapted to the situation and to the individual.

According to participants, a major impact of the project was the use of non-pharmacological interventions to manage behaviors, and the understanding that improving these behaviors takes time; there is no magical solution. As well, clinicians were better able to reframe the need to stop a behavior if it did not involve risks. Finally, the project facilitated a change in physicians' prescribing habits. Doctors said that they sometimes prescribed molecules known to have little chance of success before the project, mainly because of the pressure they felt from the staff and family to "do something ":

"One realizes very, very quickly that the medication often consists of atypical antipsychotics with an increased risk (...). And often with very, very mixed results at the expense of very important side effects. (...) but we are put in a situation where, as we have no other possible interventions, well, we have nurses at night who are few, and events happen. So, we are being asked to act quickly by saying, "Well, there is a need to do something at the medication level to decrease the behavior because it disrupts the whole structure there."

This improved management of BPSD had positives impacts on patients, families and nursing staff. Also, in cases where interventions appeared to have little impact on problematic behaviors, participants still felt that they had done everything possible through the use of teleconsultation and that it was then easier to have the staff and family accept the behavior and adapt to it. Moreover, when the staff encountered some resistance from other professionals or families, the fact that the recommendations came from a team considered to be experts increased the credibility of their recommendations.

\section{Tangible organizational repercussions}

Concrete organizational repercussions have resulted from the project on care trajectory and on the cohesion within the team. A BPSD committee was created within the MIHC to establish a clear path of care. One of the roles of this commit- tee was to receive requests from clinicians when such behaviors manifest themselves, to evaluate the patient and to establish an intervention plan, or to refer them to the tele-consultation team if needed. The members of this committee were the designated resource persons, and various clinicians in the community knew better how to proceed and whom to refer a patient. Physicians and other clinicians also felt more supported by the organization, as it provided them with the resources they needed to develop optimal clinical practices. A participant mentioned:

"Then for once, I have the impression that we are not just saying to the doctor 'well, now solve the problem'. It's a, it's a solution that involves everyone. That's a major point, a big plus."

Participants also expressed wanting to develop their autonomy in order to manage first-line and second-line cases themselves and to refer patients to the IUGM only when their own treatment plan would not successfully decrease BPSD. However, some participants feared that physicians would ignore the established care trajectory and consult directly with the IUGM team, which could have the effect of hampering the development of expertise among local teams.

\section{Important strengths of the project, despite} some remaining challenges

Described as comprehensive, available and responsive, the IUGM team inspired trust and gave hope that tele-consultations had the potential to positively impact BPSD. Participants also felt that they had enough space to stimulate and develop their skills/expertise. The presentation of recommendations in a clear, detailed report was strongly underscored as an effective strategy. Nothing was left to chance, as each issue was addressed, and the reports offered realistic and achievable recommendations.

However, some of the challenges faced by participants before the project seem to persist. For example, participants stated that some families, administrators and members of the care team persisted in their wish for a quick fix to manage BPSD, often with a "pill" to sort it out, which keeps putting pressure on clinicians. Several situations in which a suboptimal communication component has been a hindrance to the project deployment or application of the recommendations were reported as additional challenges. These situations included: (a) identifying where to forward resident files for a tele-consultation discussion; (b) failure to transmit information related to the recommendations to the persons concerned, whether it is the family doctor or inhome stakeholders and (c) transmission of information between different work shifts. 
5. The logistical organization of teleconsultations for optimal coaching benefits: divergent opinions Participants expressed a wide variety of opinions about tele-consultation planning. The main subject on which there were conflicting opinions was who should be present during tele-consultation. First, the MIHC BPSD team members felt uncomfortable when confined to a spectator role during tele-consultations for patients they did not know. However, they agreed that they have acquired knowledge when placed in these situations. Others mentioned the number of professionals present as having perhaps been too high, and they desired to limit the presence to those who are in direct contact with the patient concerned. However, the patients and their families did not seem intimidated by everyone present. Also, one participant mentioned that the presence of all clinicians, the patient, and his family was necessary because it allowed everyone to hear the same information. Non-physicians wanted the physicians to be present at tele-consultations to reassure the patient and further develop their medical expertise on BPSD. However, only a few physicians participate in tele-consultations, mainly because of time constraints, but also because of their view that the consultation report was sufficiently clear and complete, and they doubted that their presence during tele-consultations would bring additional benefits. However, they are not completely opposed to the idea. In addition, participants expressed a wish for a fixed tele-consultation schedule, in order to facilitate their attendance. Also, many wanted less frequent teleconsultations by concern that there would not be enough cases to merit monthly meetings. Participants also expressed a desire to begin discussing the cases among clinicians, in the absence of the patient and his family (who would join the meeting afterward), in order to better guide the meeting and reduce fears of saying things that would hurt a patient's or family feelings.

\section{Discussion}

The main objective of this study was to evaluate the clinical benefits and the implementation process of a tele-consultation and coaching service for BPSD. Our results support a direct benefit on patients seen in tele-consultation, with decreased symptoms and improved clinical state. Caregivers distress also diminished. The total medication prescribed decreased by $18 \%$ at 3 months. However, regarding the rationalization of antipsychotics and sedatives/hypnotics, our results were disappointing since an increase of $14 \%$ of psychotropics (mostly antipsychotics and antidepressants) was observed. This could reflect the severity of cases referred. Furthermore, the fact that there was no medical champion involved in the project and only a few doctors present for tele-consultations could also have prevented the

implementation of some pharmacological recommendations. Nonetheless, there may have been a more appropriate reorientation of prescriptions. More antidepressants were prescribed, which could reflect a better identification of anxious and depressive symptoms. Also, patients were prescribed more narcotics, which could indicate an improved recognition of pain-related BPSD. During follow-up, no hospitalization or use of restraints was necessary, which supports a better quality of care and less associated risks.

As for the implementation process, patients showed great acceptance and tolerability with the technology even when they presented severe BPSD. The presence of family members and significant staff members as well as the solemnity of the assessment, with everyone discussing one's situation, could have prevented acting-out. Also, few technical difficulties arose in conjunction with the network technology used in this project.

The local MIHC team also achieved a better understanding of each patient's problem, gained an improved sense of competency and knowledge in BPSD management. They also reported an improved ability to apply non-pharmacological recommendations. There was a high level of satisfaction with tele-consultations among the MIHC BPSD team and families. Some family members reported they would have preferred inperson consultations with specialists. However, in the context of such a remote area ( 5 hours by plane to travel from Montreal to MIHC), we suspect that tele-consultation is a good compromise.

Health professionals expressed during the interviews that this tele-consultation service represented a good solution to their isolation and helplessness. They felt supported and validated in many aspects of the care they were already giving. They described how the project allowed them to develop competencies to recognize faster BPSD, and to assess and intervene in a more comprehensive and adequate way. They felt that they were more able to implement individualized interventions in line with best practices, for example, by integrating non-pharmacological approaches instead of relying solely on medication to treat BPSD (IPA, 2015). They reported a strong desire to continue developing local expertise and they felt there were some concrete organizational repercussions from this project, with a better definition of care trajectory and also increased team unity. Both professionals and doctors felt they could rely on each other in managing these difficult cases and that they shared responsibility. They corroborated the positive impacts on patients that were measured quantitatively, and also described some benefits for families and staff. 
Similar to our results, based on 58 staff interviews, Doyle et al. (2016) reported improved outcomes for clients (symptom improvement), family and staff (reduced stress). Key themes were identified, including enhanced professional development. Interestingly, the staff also reported that the strategies they recommended held more weight with other health professionals because of the input of specialists. They also described improved collaboration and feeling part of a team, as well as reduced travel time and costs. Some difficulties with technology (sound quality and slow internet) were identified as the biggest challenge. They also identified some resistance to change, low level of staff training and high turnover, and using the medication as a "quick fix". There was, however, high satisfaction with the program's success in reducing isolation, improving confidence in staff's capacity to handle BPSD and collegial collaboration.

Our results are also consistent with other previous findings in the literature. Gentry et al. (Gentry, Lapid, \& Rummans, 2019) undertook a systematic review of geriatric telepsychiatry, including 76 papers. These studies showed feasibility and acceptability as well as high satisfaction among clients, and comparable outcomes. Ten studies describing nursing home tele-consultations were identified, also with high acceptability and satisfaction, as well as probable cost savings due to reduced travel time. One of these (Lyketsos \& Roques, 2001) also demonstrated a hospitalization rate reduced by half. Gentry et al. (Gentry, 2019) listed barriers to telemedicine that should be addressed, namely: technically challenged staff, resistance to change, quality/cost of technology, clinical factors (hearing or vision difficulties, delirium). Powers et al. (Powers, Homer, Morone, Edmonds \& Rossi, 2017) created an interdisciplinary tele-dementia clinic for rural veterans in the USA, where 95 patients were seen by different professionals, with geriatric and geriatric psychiatry diagnosis provided. Patients reported a high level of satisfaction as well as reduced travel time and costs. Medication changes were the most-frequent recommendations, and problems with rural internet connectivity were reported. A review of telemedicine and geriatrics in France (Zulfiqar et al., 2018) reported that tele-consultation in nursing homes prevents hospital transfers, optimizes treatment plan, avoids unnecessary transport, decreases healthcare expenditures and reinforces commitment by patients and families, when they are involved in the professional exchanges.

However, some challenges remain. As described by Doyle et al. (2016), the professionals described a strong persistent desire for immediate improvement of BPSD, both in the community

and in healthcare settings. This emphasized the fact that more work needs to be done in changing the philosophy of care. Every professional working in that field should be educated about dementia, BPSD, and basic care approaches. A global evaluation of every possible causes for BPSD should be advocated by staff leaders. Careful consideration for the living environment of patients with BPSD as well as staff stability should be emphasized. Comprehensive intervention plans that address causes of behaviours and includes non-pharmacological strategies instead of relying solely on medication should be advocated (IPA, 2015).

Challenges in information transmission and communication channels were also described in our study. There were divergent opinions on the logistical organization of tele-consultations in order to achieve optimal coaching benefits. The frequency of tele-consultation (fixed schedule rather than on demand) and which individuals would attend tele-consultations were matters of debate. It was difficult to reach a consensus on the optimum number of participants for providing comfort to patients and family while allowing as many professionals as possible to benefit from coaching. Some solutions could be to set up teleconsultations in a meeting room with a two-way mirror or have 2 different locations connected for the tele-consultation. This could allow as many people to attend without overwhelming the patient and his family. Furthermore, at some point during the 1-year project, it was proposed by the MIHC team that they begin tele-consultation by presenting their evaluation prior to having IUGM experts complete the information in the presence of the patient and family. This seems to correspond to one of the objectives of this project, which was to make the remote team autonomous.

Doyle et al. (2016) model implied weekly videoconferencing, telementoring (web-based team case presentations), discussions and peer support by linking regional physicians with metropolitan specialists in geriatric psychiatry. In contrast, our service allowed for direct evaluation with patients and family present. We believe this is a strength of this project as every important member of the care plan was involved and the patient was included in the discussion. Doyle et al. (2016) also reported on the importance of the family in care plan implementation. In their project, the sessions were constrained to a monthly frequency, as physicians had difficulty attending. We had the same difficulties with physician attendance in our tele-consultation service. Interdisciplinary evaluation and discussion about implementation for non-pharmacological as well as optimization of medication are strong assets that we believe improve patient's care. However, 
in order to achieve this interdisciplinary collaboration, we believe that further projects should involve medical leadership. The presence of a medical champion could be a valuable asset to ensure the transmission of pharmacological recommendations to colleagues. Even if the Alzheimer's plan requires that a medical champion be associated with BPSD teams, the reality of medical practice makes it difficult for a physician to serve as an identified coach for his colleagues. Jennings (Jennings et al. (2018) identified important issues regarding general practitioners' experiences in managing BPSD. They proposed that although an increase in knowledge would improve physician confidence, they also need to be supported by clear pathways of care and appropriate resources. Chronic nursing and orderlies understaffing acted as a barrier to implementing non-pharmacological strategies. Managers should be aware of this impact on care and be involved in creating solutions. We believe that our model of tele-consultation and coaching is an innovative way of disseminating expertise by both improving communication and collaboration between all relevant health care professionals and family members, and by building a shared team responsibility in managing these complex situations.

The small sample size of our project is a limit and it should be replicated with a larger sample to validate our results. Also, clinical data were collected on chart review, by an unblinded assistant, which could have added a bias. More research in this field is needed, with controlled trials such as the ongoing one by Piau et al. (2018) that includes quantitative and qualitative indicators as well as economic evaluation. Ideally, comparison with a controlled group (e.g. a long-term care home with usual care vs another one benefiting from the tele-consultation and mentoring 1-year service) would be indicated. Its cost-effectiveness could also be demonstrated in future research. In the future, the development of mobile devices that allow direct teleevaluation at the patient's home could increase the use of this modality, as patients and caregivers report the home setting as less distressing and more empowering (Dham et al., 2018).

\section{Conclusion}

In this article, we reported results of an evaluative research project associated with a clinical service providing tele-consultation and mentoring for BPSD. We showed positive clinical benefits for patients and demonstrated the feasibility and acceptability of its implementation. We believe that tele-consultation combined with mentoring aspects is an efficient way to provide quality of care for patients with BPSD, by promoting knowledge, expertise and skills transfer from experts to clinical staff. This type of service makes it possible to identify underlying causes of BPSD in ecological conditions and to design a personal and global plan in partnership with long-term care staff. It provides better and fairer access to specialized care.

\section{Acknowledgements}

A special acknowledgement to our partners, CAREC (Comité Aviseur pour la Recherche Clinique) and CCNA (Canadian Consortium on Neurodegeneration in Aging (Team-11)).

N. Bier is supported by a salary award from the Fonds de la recherche du Québec - Santé.

No conflict of interest was identified by the authors during this project.

\section{References}

American Geriatrics Society [AGS] 2015 Beers Criteria Update Expert Panel. (2015). American Geriatrics Society 2015 Updated Beers Criteria for Potentially Inappropriate Medication Use in Older Adults. Journal of the American Geriatric Society 63:22272246, 2015.

American Psychiatric Association. (2013). Diagnostic and statistical manual of mental disorders (5th ed.). Washington, DC: Author.

Azad, N., Amos, S., Milne, K., \& Power, B. (2012). Telemedicine in a rural memory disorder clinic-remote management of patients with dementia. Canadian Geriatrics journal, 15(4), 96-100. https://doi. org/10.5770/cgj.15.28.

Bakken, S., Grullon-Figueroa, L., Izquierdo, R., Lee, N. J., Morin, P., Palmas, W., ...Starren, J., \& IDEATel
Consortium (2006). Development, validation, and use of English and Spanish versions of the telemedicine satisfaction and usefulness questionnaire. Journal of the American Medical Informatics Association, 13(6), 660-7.

Barth, J., Nickel, F., \& Kolominsky-Rabas, P. L. (2018). Diagnosis of cognitive decline and dementia in rural areas - A scoping review. International Journal of Geriatric Psychiatry, 33(3), 459-474. https://doi. org/10.1002/gps.4841.

Bergman, H., Arcand, M., Bureau, C., Chertkow, H., Ducharme, F., Joanette, Y., \& Voyer, P. (2009). Relever le défi de la maladie d'Alzheimer et des maladies apparentées: Une vision centrée sur la personne, I'humanisme et l'excellence. Rapport du comité d'experts en vue de l'élaboration d'un plan d'action pour la maladie d'Alzheimer. http://publications.msss.gouv.qc.ca/msss/fichiers/2009/09829-01W.pdf.

Catic, A. G., Mattison, M. L., Bakaev, I., Morgan, M., Monti, S. M., \& Lipsitz, L. (2014). ECHO-AGE: an innovative model of geriatric care for long-term care residents with dementia and behavioral issues. Journal of the American Medical Directors Association, 15(12), 938-942. https://doi.org/10.1016/j. jamda.2014.08.014 
Creswell, J. W. \& Plano Clark, V. L. (2007). Designing and conducting mixed methods research. Thousand Oaks, CA: Sage Publications.

Cummings, J. L. (1997). The Neuropsychiatric Inventory: assessing psychopathology in dementia patients. Neurology, 48(5 Suppl 6), S10-16.

Dang, S., Gomez-Orozco, C. A., van Zuilen, M. H., \& Levis, S. (2018). Providing Dementia Consultations to Veterans Using Clinical Video Telehealth: Results from a Clinical Demonstration Project. Telemedicine Journal and E Health, 24(3), 203-209. https://doi.org/https://doi:10.1089/tmj.2017.0089

Dham, P., Gupta, N., Alexander, J., Black, W., Rajji, T., \& Skinner, E. (2018). Community based telepsychiatry service for older adults residing in a rural and remote region- utilization pattern and satisfaction among stakeholders. British Medical College of Psychiatry, 18(1), 316. https://doi.org/10.1186/ s12888-018-1896-3

Doyle, C., Jackson, D., Loi, S., Malta, S., \& Moore, K. (2016). Videoconferencing and telementoring about dementia care: evaluation of a pilot model for sharing scarce old age psychiatry resources. International Psychogeriaticsr, 28(9), 1567-1574. https://doi.org/10.1017/s1041610216000740

Fernández, M., Gobartt, A. L., Balañá, M., \& COOPERA Study Group (2010). Behavioural symptoms in patients with Alzheimer's disease and their association with cognitive impairment. British Medical College of neurology, 10, 87. https://doi. org/10.1186/1471-2377-10-87

Gauthier, S., Cummings, J., Ballard, C., Brodaty, H., Grossberg, G., Robert, P., \& Lyketsos, C. (2010). Management of behavioral problems in Alzheimer's disease. International Psychogeriatrics, 22(3), 346372. https://doi.org/10.1017/s1041610209991505

Gentry, M. T., Lapid, M. I., \& Rummans, T. A. (2019). Geriatric Telepsychiatry: Systematic Review and Policy Considerations. American Journal of Geriatric Psychiatry, 27(2), 109-127.

Gordon, S. E., Dufour, A. B., Monti, S. M., Mattison, M. L., Catic, A. G., Thomas, C. P., \& Lipsitz, L. A. (2016). Impact of a Videoconference Educational Intervention on Physical Restraint and Antipsychotic Use in Nursing Homes: Results From the ECHOAGE Pilot Study. Journal of the American Medical Director Association, 17(6), 553-556. https://doi. org/10.1016/j.jamda.2016.03.002

Guy, W. (1976). ECDEU Assessment Manual for Psychopharmacology. Rockville,MD, U.S.: Department of Health, Education, and Welfare.

Hurt, C., Bhattacharyya, S., Burns, A., Camus, V., Liperoti, R., Marriott, A., Byrne, E.J. (2008). Patient and Caregiver Perspectives of Quality of Life in Dementia. Dementia and Geriatric Cognitive Disorders 26:138-146.

Institut de la statistique du Québec. (2016). Le bilan démographique du Québec. http://www.stat.gouv. qc.ca/statistiques/population-demographie/bilan2016.pdf

International Psychogeriatric Association. (2015). The IPA Complete Guides to BPSD - Specialists Guide. https://www.ipa-online.org/publications/guidesto-bpsd.
Jennings, A.A., Foley, T., Walsh K.A., Coffey, A. Browne, J.P., \& Bradley, C., P. (2018). General practitioners' knowledge, attitudes, and experiences of managing behavioural and psychological symptoms of dementia: A mixed-methods systematic review. International Journal of Geriatric Psychiatry, 33:1163-1176.

Lee, J.H., Kim, J.H., Jhoo, J.H., Lee, K.U., Kim, K.W., Lee, D.Y., \& Woo, J.I. (2000). A Telemedicine System As a Care Modality for Dementia Patients in Korea. Alzheimer Disease and Associated Disorders 14(2): 94-101.

Loh, P.K., \& Donaldson, M. (2007). Development of a telemedicine protocol for the diagnosis of $\mathrm{Alz}$ heimer's disease. Journal of Telemedicine and Telecare, 13(2), 90-94.

Luppa, M., Luck, T., Brahler, E., Konig, H. H., \& RiedelHeller, S. G. (2008). Prediction of institutionalisation in dementia. A systematic review. Dementia and Geriatric Cognitive Disorders, 26(1), 65-78. https://doi.org/10.1159/000144027.

Lyketsos C.G., \& Roques C. (2001). Telemedicine use and the reduction of psychiatric admissions from long-term care facility. Journal of Geriatry Psychiatry and Neurology, 14(2), 76-79.

Ministère de la Santé et des Services Sociaux, Institut national de la statistique de la province de Québec, (2016). Maladie d'Alzheimer et autres troubles neurocognitifs majeurs : portrait territorial. Document de travail.

Miles, M.B., \& Huberman, M.A. (2003). Analyse des données qualitatives, 2ème edition Paris: De Broeck.

Morgan, D. G., Crossley, M., Kirk, A., D'Arcy, C., Stewart, N., Biem, J., McBain, L. (2009). Improving access to dementia care: development and evaluation of a rural and remote memory clinic. Aging and Mental Health, 13(1), 17-30. https://doi. org/10.1080/13607860802154432.

Murman, D. L., \& Colenda, C. C. (2005). The economic impact of neuropsychiatric symptoms in Alzheimer's disease: can drugs ease the burden? Pharmacoeconomics, 23(3), 227-242. https://doi. org/10.2165/00019053-200523030-00004.

Okura, T., Plassman, B. L., Steffens, D. C., Llewellyn, D. J., Potter, G. G., \& Langa, K. M. (2010). Prevalence of neuropsychiatric symptoms and their association with functional limitations in older adults in the United States: the aging, demographics, and memory study. Journal of the American Geriatric Society, 58(2), 330-337. https://doi.org/10.1111 /j.1532-5415.2009.02680.

Ornstein, K., \& Gaugler, J. E. (2012). The problem with "problem behaviors": a systematic review of the association between individual patient behavioral and psychological symptoms and caregiver depression and burden within the dementia patient-caregiver dyad. International Psychogeriatrics, 24(10), 1536-1552. https://doi.org/10.1017/ S1041610212000737.

Piau, A., Nourhashemi, F., De Mauleon, A., Tchalla, A., Vautier, C., Vellas, B. Soto Martin, M. (2018). Telemedicine for the management of neuropsychiatric symptoms in long-term care facilities: the DETECT study, methods of a cluster randomised controlled trial to assess feasibility. British Medical Journal 
Open, 8(6), e020982. https://doi.org/10.1136/bmjopen-2017-020982.

Powers, B. B., Homer, M. C., Morone, N., Edmonds, N., \& Rossi, M. I. (2017). Creation of an Interprofessional Teledementia Clinic for Rural Veterans: Preliminary Data. Journal of the American Geriatric Society, 65(5), 1092-1099. https://doi.org/10.1111/ jgs.14839.

Rabinowitz, T., Murphy, K. M., Amour, J. L., Ricci, M. A., Caputo, M. P., \& Newhouse, P. A. (2010). Benefits of a telepsychiatry consultation service for rural nursing home residents. Telemedicine Journal and E Health, 16(1), 34-40. https://doi.org/10.1089/ tmj.2009.0088.

Ramos-Rios, R., Mateos, R., Lojo, D., Conn, D. K., \& Patterson, T. (2012). Telepsychogeriatrics: a new horizon in the care of mental health problems in the elderly. International Psychogeriatrics, 24(11), 1708-1724. https://doi.org/10.1017/ s1041610212000981.

Rechel, B; Dakula, A; Duran, A; Fattore, G; Edwards, N; Grignon, M; Smith, TA (2016) Hospitals in rural or remote areas: An exploratory review of policies in 8 high-income countries. Health Policy,
120, 758-769. https://doi.org/10.1016/j.healthpol.2016.05.011.

Saligari, J., \& Flicker, L. (2002). The clinical achievements of a geriatric telehealth project in its first year. Journal of Telemedicine \& Telecare, 8 (S3), 53-55.

Shulman, B., Conn, D., \& Elford, R. (2006). Geriatric telepsychiatry and telemedicine: a literature review. Canadian Journal of Geriatrics, 9, 139-146.

Steinberg, S. I. (2014). Telepsychiatry for geriatric residents in rural nursing homes. Alzheimer's \& Dementia. The Journal of the Alzheimer's Association, 10(4), 768.

Wadsworth, H. E., Dhima, K., Womack, K. B., Hart, J., Jr., Weiner, M. F., Hynan, L. S., \& Cullum, C. M. (2018). Validity of Teleneuropsychological Assessment in Older Patients with Cognitive Disorders. Archives of Clinical Neuropsychology, 33(8), 1040 1045. https://doi.org/10.1093/arclin/acx140.

Zulfiqar, A. A., Hajjam, A., Talha, S., Hajjam, M., Hajjam, J., Erve, S., \& Andres, E. (2018). Telemedicine and Geriatrics in France: Inventory of Experiments. Current Gerontology and Geriatrics Research, 2018, Article ID 9042180, 10 pages. https://doi. org/10.1155/2018/9042180. 